\title{
MRI Compatible Modular Designed Robot for Interventional Navigation - Prototype Development and Evaluation -
}

\author{
Hiroaki Naganou $^{1}$, Hiroshi Iseki ${ }^{2}$, and Ken Masamune ${ }^{1}$ \\ ${ }^{1}$ Tokyo Denki University, Ishizaka, Hatoyama-cho, Hiki, Saitama \\ ${ }^{2}$ Tokyo Women's Medical University, Kawada-cho8-1, Shinjyuku-ku ,Tokyo
}

\begin{abstract}
Interventional MRI therapy has started in these years, and many researchers are focusing on surgical robots operated under the MRI environment to achieve most effective image guided surgery. In this paper, the prototype development of MRI compatible modular-designed navigation robot is proposed as the basic components of the future MRI-guided robot surgery. System features and the evaluation testing are described
\end{abstract}

\section{Introduction}

The surgical procedures that perform drilling such as a biopsy or inject drugs, requires accurate positioning, and in case of interventional MRI therapy, engineering technologies are considered useful to give surgeon precise orientation information to reach to the target tumor more precisely [1]. In this research, we proposed and developed the prototype of simple two D.O.F. robot for needle guidance surgery, that characteristics are followings: intended to use inside the MRI gantry, modular design for apply many kinds of surgical procedure, well-consideration of sterilization, considering usability with 2DOF active arm and 14 DOF passive arm which materials are MRI-compatible. We describe the design strategy, and the evaluation testing of the image distortion caused by the robot.

\section{Description of the Robot Design}

To support navigation surgery, we decided to give only two DOF, that are indispensable for drilling. The robot performs only positioning of an angle, and compensate surgeon's hand tremor. The developed robot is shown in a Fig.1. The robot is composed of a linear actuator and a rotary actuator. It has two arms and small free joints on the arms' tip for guiding a needle. A surgeon locates the arm 1 at the insertion point, then the insertion angle is fixed by arm 2 which is moved by the actuators. Finally, the surgeon punctures the needle by himself. A cleanliness of the mechatronics part can be maintained by sterilization cover sheet. Instead of using a ferromagnetic metal, we adopted the non-ferro magnetic metal, the reinforced plastic, and the synthetic resin. The ultrasonic motor (Shinsei Kogyo, Japan) was adopted as an actuator. 


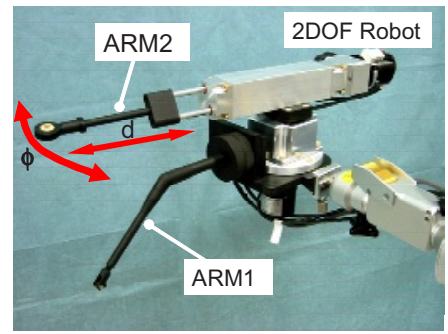

Fig. 1. MRI compatible modular designed robot for interventional navigation
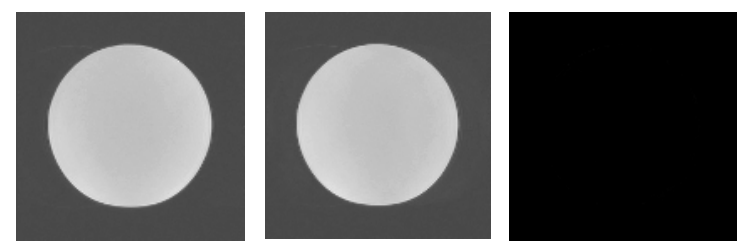

Fig. 2. From left to right: MR-image when nstalling robot, Control image, Subtraction image

\section{Experiments, Results, and Discussion}

Mechanical accuracy evaluation : Backlash, positioning accuracy and repeatability are measured subject to JIS standard method. The backlash was $0.37[\mathrm{~mm}]$, the positioning accuracy $0.14[\mathrm{~mm}]$, and the repeatability $\pm 0.085[\mathrm{~mm}]$. Evaluation about a rotation mechanism has not been carried out.

Image distortion study : To evaluate the influence of the navigation robot installed into MR gantry, we evaluated the image distortion with the cylindrical phantom filled with MR-enhance solution, which is used for the initial set-up calibration of the MRI system. We calculated the subtraction image to evaluate noise caused by the manipulator. The subtraction image was calculated from the image with a robot, and the image without installation (Fig. 2.).

Discussion: Considering the MR image resolution of $1 \mathrm{~mm}$, the results of mechanical accuracy was enough as the surgical robot for needle guidance surgery. We calculated the Signal-to-Noise ratio (It defines as the ratio of average of pixel intensity of ROI and standard deviation of the noise area) of an images, in order to evaluate the noise caused by the navigation robot. $\mathrm{S} / \mathrm{N}$ ration of MR-image when installing robot is 108.65. S/N ration of Control image is 153.21 . From here onwards, we can evaluate the quality of image which is debased by installed robot.

\section{Conclusion}

In this study, 2-DOF MRI compatible modular designed navigation robot is proposed and the fundamental evaluations are performed. This robot will be useful for the MRI image guided therapy performed inside the MRI gantry in the future.

\section{References}

1. K Masamune, et al.: Development of an MRI compatible Needle Insertion Manipulator for Stereotactic Neurosurgery, J Image Guided Surgery, Vol.1, pp.242-248, 1995 